\title{
Association of electronic cigarette usage and nicotine consumption frequency of young adults in British Columbia
}

\author{
Adora Kwong ${ }^{1}$, Dale Chen ${ }^{2}$ \\ 1. Lead author, B. Tech student, School of Health Sciences, British Columbia, Institute of Technology, 3700 Willingdon Ave, Burnaby, BC V5G 3 H2 \\ 2. Supervisor, School of Health Sciences, British Columbia Institute of Technology, 3700 Willingdon Ave, Burnaby, BC V5G $3 \mathrm{H} 2$
}

\section{Abstract}

\section{Background}

Electronic e-cigarette ever users has been increasing as of 2015, the most prevalent ever users being young adults aged 20-24 years old. The implication of e-cigarette ever user developing into long term users is a emerging public health concern.

\section{Methods}

Electronic cigarette usage frequency and nicotine consumption was measured through a selfadministered online survey of young adults $(n=54)$. Survey was advertised through social media sites between January 2019 till February 2019. Descriptive and inferential statistic was conducted using NCSS 12 to examine the association between electronic cigarette usage and nicotine consumption.

\section{Results}

Among young adults aged 19 to 24 years old, the frequency of e-cigarette usage was $51 \%$ high usage, $31 \%$ no usage and $16 \%$ medium and low usage. For nicotine consumption, respondents were $25 \%$ daily, $40 \%$ no use, $18 \%$ infrequent, and $14.8 \%$ frequent.

\section{Conclusion}

There is an association between more frequent electronic cigarette usage and higher nicotine consumption among young adults in British Columbia. Frequency e-cigarette users were found to consumption nicotine at higher frequency then non users. Further research is needed to fully understand the extent of the relationship of if e-cigarette usage promotes daily nicotine consumption or daily nicotine consumption results in higher e-cigarette usage.

Key words: Electronic cigarette, E-cigarettes, nicotine, young adults, British Columbia

\section{Introduction}

Electronic-cigarettes (e-cigarettes) defined in British Columbia as a product or device which contains an electric heating element capable of vaporizing an esubstance for inhalation or aerosolization (1). E-cigarettes use a solution which fills a 
cartridge within the device to be vaporized. This solution can contain tobacco with bases such as propylene glycol, and flavoring(2). This literature reviews will focus on tobaccobased E-cigarette as there are liquids on the market that do not contain tobacco among other various substances. In 2015, Canadian over 15 years old reported that 3.2\% (3.9 million) of them having ever tried an ecigarette(3). The same report also noted a significant increase between 2013 and 2015 of use within 30 days of their survey. This growing trend of use of e-cigarettes has prompted research in all areas of e-cigarette particularly in the motivation of use. Previous research done on adults have found that smoking cessation was the main motivation for e-cigarette uses (4). Though these studies have found a correlation with smoking cessation and e-cigarette use among older user of e-cigarettes there is less knowledge on young adult use.

The study comes during the growing popularity of e-cigarette usage. The recent FDA investigation on JUUL, a popular ecigarette producer, regarding attractive adolescent smokers and subsequent shut down of their flavoured products has brought the topic of e-cigarettes and adolescents to the forefront of public health. The study will examine the pattern of usage of e-cigarettes use among young adults in $\mathrm{BC}$ and to explore if the use of e-cigarettes in young adults could potentially be correlated to long-term usage.

\section{Literature review}

Current E-cigarette uses in Canada

According to the Tobacco Use Canada Report done by the University of Waterloo estimates that $13.0 \%$ of Canadian are smokers, the lowest prevalence estimates since monitoring began (3). Of those smokers, the report found that $13.2 \%$ (3.9 million) have tried an e-cigarette once in their lifetime and 1.0\% ( 308 000) reporting daily use as of 2015. With growing numbers of the user through 2015 to 2017. In addition, ecigarette use was found to be most prevalent among young people, three-tenths of young adults aged 20-24 reported trying ecigarettes while one-fourth youth aged 15-19 reported trying e-cigarettes. Prevalence was also greater among smokers at $51.0 \%$ versus non-smokers at $7.6 \%$. The report also states that adults aged 45 and older are more prevalent of being daily users of those who used e-cigarettes (3). E-cigarette use motivations are varied. Many markets have advertised electronic cigarette to help smokers with smoking cessation. Though research has found motives for e-cigarette use among various age groups differ.

\section{The motivation for use among adults}

An online survey was done in by Volesky et al. in which individuals that purchased from the 17 e-cigarettes shops located in Ottawa could participate, had most respondents that had a history of smoking or have previous experience with e-cigarette products. The median age of the survey was 38.1 years old. Those respondents were found to have various reasons for initiation of 
e-cigarettes, with more than half of respondent indicating that there was hoped to stop using e-cigarettes at some point in the future. Many respondents indicated a desire for reducing or eliminating cigarette use as a very important reason for initiation(5). This idea that e-cigarette use for the cessation of smoking isn't novel but has been supported by anecdotal evidence throughout the beginning of e-cigarette popularity with many marketing companies strive to promote that benefit. The study done by Volesky et al. further shows that among adult users there is a some desire for smoking cessation when using ecigarettes(5).

\section{E-cigarettes for smoking cessation}

Many individuals that seek tobacco cessation turn to various tools. One of which that has gained recent popularity is electronic cigarettes. Marketers have been advertising the benefits of using electronic cigarettes for cessation purposes since there commercialization in the early 2000's. This claim has prompted many researchers to test their validity. Many agree on the population health benefit if all conventional smokers switched to e-cigarettes due to the vapourization of e-liquid as opposed to the combustion of tobacco avoiding generation of toxic combustion products(6). Though the efficacy of e-cigarette for smoking cessation is not well established with conflicting reports. A longitudinal self-reported survey was done in 2013 on current and former smokers in Canada, US, UK, and Australia through a 12-month period. Researchers found that electronic cigarette users were no more likely to quit smoking(7). The study was limited due to its design, in which it did not focus on the effect of e-cigarette on cessation. Grana et al., 2014 examined US smokers in 2014 through a longitudinal selfreported survey over 12 months sought to determine if e-cigarettes use predicted cessation or reduced cigarette consumption. The study found no significant predictability of quitting after 1 year or reduction of cigarette usage over 12 months(8). This study failed in regards of having controls and information of e-cigarette use as in frequency, duration, and pattern of use. Both longitudinal studies were limited as they were both self-reported surveys thus not capturing the full breadth of individuals that may have succeeded in smoking cessation. Individuals that succeeded in smoking cessation mostly were no longer as interested in the study and would self exclude themselves. Even with these limitations, both longitudinal studies seem to suggest no correlation between smoking cessation and e-cigarettes. A study in 2016 by Zhuang et al. found through a 2-year survey of 2028 US smoker that the previous study mentioned findings may not have fully illustrated the benefits of e-cigarettes with cessation(9). Through a 2-year follow up survey they found that long-term ecigarettes use was associated with higher quit attempt rate than short-term use $(72.6 \%$ vs $53.8 \%$ ) (9). Short-term use was defined as only using e-cigarette at baseline or at follow up and long-term use was using e-cigarette at baseline and at follow. This study illustrates a 
potential of e-cigarettes as a long-term smoking cessation tool as it spans for 2 years instead of 1 as the previous two studies.

The motivation for use among youth and young adults

The Tobacco Use Canada reported that the most prevalent age group to have tried e-cigarettes are aged 15-19 years old. Researchers have proposed various models for e-cigarette attractiveness among adolescents ranging from perceived health risks, specific product characteristics (such as taste, price and inconspicuous use), and higher levels of acceptance among peers(10). A survey done on ninth grade students in the Niagara region found that among ninth-grade students smoking reduction and cessation was not a motivation factors for their use of e-cigarettes(11). The respondents appear to be motivated by the novelty of trying something new (11). This difference in motivation of e-cigarette use is carried through young adults also. Researchers surveyed 4444 students from 8 colleges in North Carolina in a web-based survey in fall 2009(12). The median age of this study is 20.5, they found that e-cigarette use was not associated with intention of smoking cessation. Unlike the survey done by Volesky et al. where the median age was higher at 38.1, the younger population who use ecigarettes are motivated by other reason. A cross-sectional study on adult smokers in Barcelona suggested that young users, aged 25 and younger use of e-cigarettes are more likely to be associated with the flavours other than tobacco(13). Prompting the suggestion that the flavours of e-cigarette create a novel experience that entices adolescences and young adults.

The surveys conducted by Bauld et al. in the United Kingdom through 2015 -2017 found that that around a tenth to a fifth of 11-16-year-old report having tried ecigarettes, only $3 \%$ or less report using them at least weekly(14). Thus, putting the hypothesis that experimentation with ecigarettes does not necessarily translate into regular use, particularly among never smokers for now in UK (14). This may provide some relief for the concern that E-cigarette use could be a gateway to youth smoking.

\section{Susceptibility of smoking}

While in Canada conventional smoking prevalence among the youngest population was relatively low, students may be susceptible to future smoking. This is defined by "the absence of a firm decision not to smoke,"(15) In Hong Kong, researchers have found that favorable perceptions through parental and school acceptability lead adolescences to become more susceptible(16). Furthermore, this susceptibility to smoking can be exasperated by various personal, social and environmental factors among high school children (17).

Adolescents are more likely to initiate combustible tobacco use if they have been ever users of e-cigarettes(18). In Los Angeles 2530 high schoolers age 14 years were examined through a self-reported longitudinal repeated assessment of a school-based cohort at baseline, 6-month 
follow-up and 12- month follow-up. They found that ever users of e-cigarette compared to nonusers were more likely to report initiation of combustible tobacco use such as cigarettes, cigars, and hookah over the next year(18).

The susceptibility of smoking among adolescences and young adults is important because researchers have found that initiation of smoking at an earlier age will increases one's risk of smoking later. Gagne et al found that not only is that true for youths, where a large proportion of those that start as young adults will have a high likelihood of becoming regular smokers. Furthermore, they suggest that even light and intermittent smoking among young adults have a higher risk of upgrading to daily smoking, morbidity, and mortality in adulthood(19). Through a Monte Carlo stochastic simulation model developed to asses the balance of health benefits and harms of e-cigarettes use at the population, analysis has estimated the increased population-level harm if e-cigarettes use among adolescent and young adults confers to long term usage(20).

\section{Material}

Computer software was used to collect and analyze data. SurveyMonkey was used to administer the online survey and for descriptive statistic. NCSS 12 was used to analyze inferential statistic.

\section{Methods}

Participants
Participants were individuals that are adults aged 18 to 24 years old residing in British Columbia at the completion of the survey. Individuals aged 15 and above are included in the study as they can read the consent form and make an informed decision for themselves though their answers will not be analyzed. Individuals older than 25 have been excluded due to the focus of the study is on the younger population. Ethnicity will not be differentiated, as age is our focus.

Participants in this study were on a voluntary basis and were not be compensated.

\section{Measure}

E-cigarette usage

E-cigarette usage was measured through a multichotomous for the past 30 days ranging from no use (none), single use, 1-5 times (low), 5-10 times (medium) and, $10+$ times (high).

\section{Nicotine Consumption}

Nicotine consumption was measured through a multichotomous ordinal scale from daily, frequent (3-6 times a week), infrequent (1-8 times a month), single use (tried once) and, never. Concentration of nicotine consumption per day is not asked, just the frequency at which nicotine is consumed. Procedure

Survey was released on January 29, 2019 and closed on February 13 $3^{\text {th }}, 2019$ on Instagram through a public personal account with the hashtags public health, Vancity, $\mathrm{BCIT}, \mathrm{BC}$, ecigarette, and vaping. The survey was released on Reddit under the sub thread 
of publichealth, Vancouver, BCIT, BC, electronic_cigarette, Canadian_ecigs, and vaping101.

\section{Results}

Demographic breakdown of respondent was split 24 women and 30 men. Among the 54 young adults aged 19 to 24 years old, the frequency of e-cigarette usage was $51 \%$ for high usage (28), 31\% no usage (17), $14 \%$ medium (8), and 2\% low usage (1). For nicotine consumption, respondents were $40 \%$ no use (22), $25 \%$ daily (14), $14.8 \%$ infrequent (8), 14.8\% high frequency (8) and, $3.7 \%$ single (2).

\section{Inferential statistics}

Chi-square test was performed on the data to examine the association between ecigarette use and nicotine consumption. The study set the significance level at 95\% ( $p>0.05)$.

The study found that there was an association between e-cigarette use frequency and nicotine consumption among adults aged $18-24$ years in $B C(P=0.000)$.

\section{Chi-square test was also run} between participants that answered zero use and single use of e-cigarettes compared to 1-5 times, 5-10 times and, 10+ times e- cigarette usage. This analysis was done as zero and single users can categorize as trial users. This analysis also concluded that there is an association between e-cigarette users and nicotine consumption among adults aged 18 -24years in $\mathrm{BC}(\mathrm{P}=0.000)$.

\section{Discussion}

The data provide evidence that ecigarette use is significantly associated with increased nicotine consumption for young adults. The study found that the participants $(n=54)$ had an association between increased nicotine consumption and e-cigarette use frequency $(P=0.000)$. Young adults with a higher frequency of e-cigarette user have a higher likelihood of consuming more nicotine. This is concerning because researchers have shown that the younger individuals start smoking the longer, they will smoke in their lifetime (19).

When the frequency of use was separated into two categories, non-users those that answered zero and single use of ecigarettes was compared to e-cigarette users which comprised of the remaining categories. The study found that non-users were more likely to consume less nicotine

Table 1. Frequency of e-cigarette usage and nicotine consumption contingency table

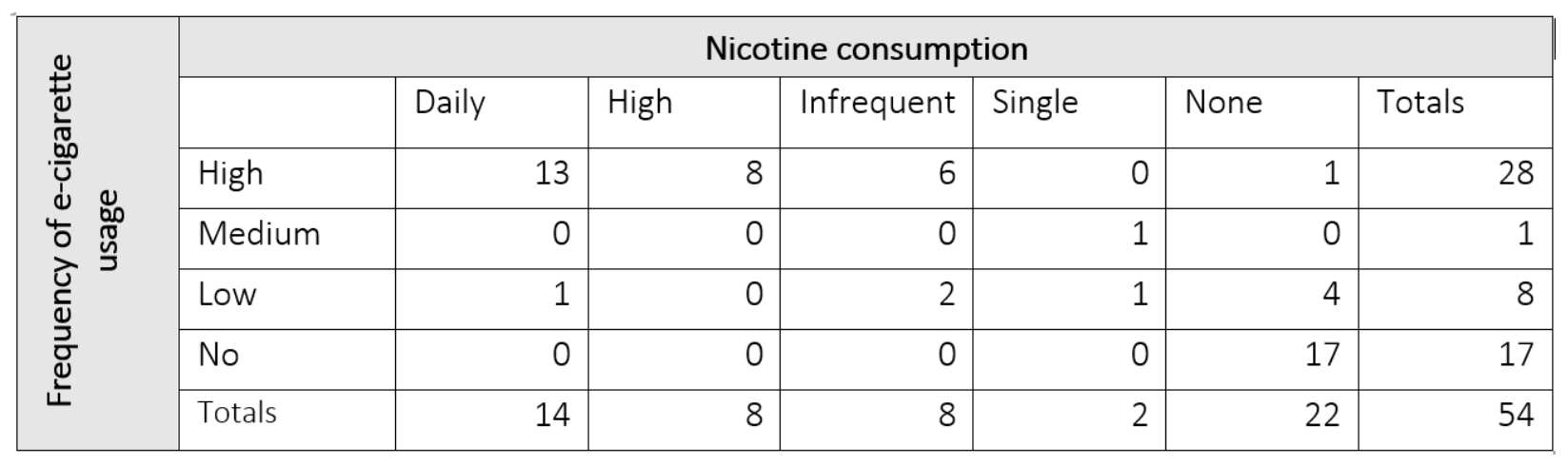


compared to e-cigarette users $(P=0.000)$. This was seen in the UK as experimentation with e-cigarettes did not translate into regular use of tobacco products(14). The results further support the research done in LA where high schoolers who were ever users of e-cigarettes were more likely to initiate combustible tobacco products such as cigarettes, cigar, and hookah which all contain nicotine (18). As e-cigarette usage is a relatively new topic particularly in younger users such as adolescents and young adults, there is little research available currently that this research refutes.

\section{Knowledge translation}

Though research focused on young adults, there could be future public health initiatives to prevent e-cigarette usage in youths and young adults. As of 2016, ecigarette and vapour products have been included in the BC Tobacco and Vapour Products Control Act which prohibits the purchase for minors.

This study provides evidence that there is an association between e-cigarette usage and nicotine consumption, but high level of e-cigarette is mainly restricted to frequent nicotine consumers. There isn't evidence that non users of e-cigarettes consume nicotine regularly. Population health information exchange to guardians of youth can be focused towards looking for long term patterns of use of e-cigarettes as a potential for smoking initiation. Potentially guidance documents can assist guardians and parents on risk assessment for youths and young adults with e-cigarette usage.

\section{Limitation}

The focus of the study is on young adults age 18 to 24-year-olds. Only a selfadministered survey online was used to gather data, individuals were thus selfselecting. Individuals self selected through their participation in various subreddit selected and social media activity on Instagram during the research. A combination of methodology from in-person and online would provide a better sampling frame. Not all young adults will be online at the specific subreddits chosen. The study would be more representative if in-person surveys were conducted in addition to an online survey at locations where young adults congregate such as post-secondary school, malls, gyms, etc. Due to the age group of focus, this limitation may not be as exaggerated as many young adults will frequent social media. A combination of survey method could increase external validity and lower beta error.

The study was limited for time as the survey was released on January 29th 2019 and closed on February 13 $3^{\text {th }}, 2019$. Time limitation was due to the structure of the course schedule and the required ethics board approval. Subsequent research could extend the length of the survey to capture more respondents. It would be reasonable for future research to survey for a month or up to 3 months. This could improve external validity. 
The amount of nicotine consumed was not measured. This was due to the complexity of correctly assessing nicotine consumption through a self-administered survey. Nicotine consumers may be aware of consumption but not the amount. Users are unlikely to calculate their exact amount daily or weekly. Various brands of nicotine products will have varying nicotine concentration. The concentration of nicotine may not be clearly or properly labeled in certain products. This unknown of the amount of nicotine consumption interferes with the internal validity of the research as there is likely a behavioural difference between those that consume high amounts of nicotine as opposed to low consumers. As dependence on nicotine could be a factor in e-cigarette usage.

\section{Future research}

1. E-cigarette usage and cannabis usage should be investigated with the legalization of Cannabis. Investigating the role of e-cigarettes with cannabis consumption as one of the active ingredients in cannabis THC can be incorporated into e-liquids. There could be increase of e-cigarette usage for cannabis consumption.

2. E-cigarette initiation motivation among youths and young adults. This topic hasn't been fully explored in current literature particularly in Canada. Most research has been exploring the use of e-cigarette as a smoking cessation tool. Some research has suggested the novelty of e-cigarettes as a motivator for younger consumers.

3. E-cigarette usage and concentration of nicotine consumption. This study focused on the frequency of nicotine consumption, but concentration would be a more quantitative assessment of the association between e-cigarette usage and nicotine consumption. Nicotine quantity could provide evidence on the use of e-cigarette as a smoking cessation tool.

\section{Conclusion}

Among young adults in British Columbia, those that have more e-cigarette use are associated with higher nicotine consumption. Frequent e-cigarette users are positively associated with higher monthly nicotine consumption as opposed to nonfrequent e-cigarette users. This evidence may give relief to the concern of the evergrowing number of youths that have ever tried e-cigarette. This study shows that regular nicotine consumption is mostly restricted to regular e-cigarette users and not ever users. This evidence suggests that population health concerning nicotine addiction and smoking prevention should focus itself with increased e-cigarette usage frequency among individuals and less on ever users.

\section{Acknowledgement}


I would like to express my deepest gratitude to my research supervisor, Dale Chen, for his ongoing support, patience and constructive feedbacks throughout the duration of my project. Acknowledgement is also warranted for Dr. Allison Kirschenmann, for her expertise and help for the ethics approval of my survey. Lastly to Dr. Helen Heacock for her aid and guidance during the beginning stages and development of my study. 


\section{References}

1. Tobacco and Vapour Products Control Act. CHAPTER 451; 1996.

2. Brazier Y, Biggers, Alana MD M. Are ecigarettes a safe alternative to smoking? Medical News Today. 2018 Jun;

3. Jessica Reid, David Hammond, Vicki Rynard, Cheryl Madill RB. Tobacco Use in Canada: Patterns and Trends 2017 Edition | ECTA of Canada. 2017.

4. Kaspick S. Electronic cigarettes: What motivates people to use them? BCIT Environ Heal J. 2017 May;

5. Volesky KD, Maki A, Scherf C, Watson LM, Cassol E, Villeneuve PJ. Characteristics of e-cigarette users and their perceptions of the benefits, harms and risks of ecigarette use: survey results from a convenience sample in Ottawa, Canada.

6. Benowitz NL. Emerging Nicotine Delivery Products. Implications for Public Health. Ann Am Thorac Soc [Internet]. 2014 Feb 27 [cited 2019 Apr 10];11(2):231-5. Available from: http://www.atsjournals.org/doi/abs/10.1 513/AnnalsATS.201312-433PS

7. Lee AHY, Stater BJ, Close L, Rahmati R. Are e-cigarettes effective in smoking cessation? Laryngoscope. 2015 Apr;125(4):785-7.

8. Grana RA, Popova L, Ling PM. A longitudinal analysis of electronic cigarette use and smoking cessation. JAMA Intern Med. 2014 May;174(5):8123.

9. Zhuang Y-L, Cummins SE, Y Sun J, Zhu S$\mathrm{H}$. Long-term e-cigarette use and smoking cessation: a longitudinal study with US population. Tob Control. 2016 Oct;25(Suppl 1):i90 LP-i95.

10. Schneider S, Diehl K. Vaping as a Catalyst for Smoking? An Initial Model on the Initiation of Electronic Cigarette Use and the Transition to Tobacco Smoking Among Adolescents. Nicotine Tob Res [Internet]. 2016 May [cited 2019 Apr 10];18(5):647-53. Available from: https://academic.oup.com/ntr/articlelookup/doi/10.1093/ntr/ntv193

11. Khoury M, Manlhiot C, Fan C-PS, Gibson D, Stearne K, Chahal N, et al. Reported electronic cigarette use among adolescents in the Niagara region of Ontario. Can Med Assoc J. 2016 Aug;188(11):794-800.

12. Sutfin EL, McCoy TP, Morrell HER, Hoeppner BB, Wolfson M. Electronic cigarette use by college students. Drug Alcohol Depend. 2013 Aug;131(3):21421.

13. Bunch $K$, Fu M, Ballbè $M$, MatillaSantader N, Lidón-Moyano C, MartinSanchez JC, et al. Motivation and main flavour of use, use with nicotine and dual use of electronic cigarettes in Barcelona, Spain: a cross-sectional study. BMJ Open. 2018 Mar;8(3):e018329.

14. Bauld L, MacKintosh AM, Eastwood B, Ford A, Moore G, Dockrell M, et al. Young People's Use of E-Cigarettes across the United Kingdom: Findings from Five Surveys 2015-2017. Int J Environ Res Public Health. 2017 Aug;14(9).

15. Hammond D, Reid JL, Cole AG, Leatherdale ST. Electronic cigarette use and smoking initiation among youth: a longitudinal cohort study. CMAJ. 2017 Oct;189(43):E1328-36.

16. Leung L, Ho S, Chen J, Wang M, Lam T. Favourable Perceptions of Electronic Cigarettes Relative to Cigarettes and the Associations with Susceptibility to Electronic Cigarette Use in Hong Kong Chinese Adolescents. Int J Environ Res Public Health. 2018 Jan;15(1):54. 
17. Polańska K, Wojtysiak P, Bąk-Romaniszyn L, Kaleta D. Susceptibility to cigarette smoking among secondary and high school students from a socially disadvantaged rural area in Poland. Tob Induc Dis. 2016 Aug;14:28.

18. Leventhal AM, Strong DR, Kirkpatrick MG, Unger JB, Sussman S, Riggs NR, et al. Association of Electronic Cigarette Use With Initiation of Combustible Tobacco Product Smoking in Early Adolescence. JAMA [Internet]. 2015 Aug 18 [cited 2019 Mar 16];314(7):700. Available from: http://jama.jamanetwork.com/article.asp x?doi=10.1001/jama.2015.8950

19. Gagné MSc T, Veenstra PhD G. Trends in smoking initiation in Canada: Does noninclusion of young adults in tobacco control strategies represent a missed opportunity? Can J Public Heal. 2017;108(1):E14-20.

20. Warner KE, Mendez D. E-cigarettes: Comparing the Possible Risks of Increasing Smoking Initiation with the Potential Benefits of Increasing Smoking Cessation. Nicotine Tob Res [Internet]. 2019 Jan 1 [cited 2019 Apr 10];21(1):417. Available from:

https://academic.oup.com/ntr/article/21 /1/41/4956222 